\title{
ACHIEVING BETTER PATIENT CARE THROUGH CLINICO-PATHOLOGIC RELATIONSHIP BETWEEN FORENSIC MEDICINE AND OTHER CLINICAL DISCIPLINES
}

\author{
Hulathduwa SR. Ariyarathne D.
}

\begin{abstract}
Senior Lecturers, Dept. of Forensic Medicine, Faculty of Medical Sciences, University of Sri Jayawardenepure, Sri Lanka
\end{abstract}

\begin{abstract}
No branch or sub-specialty of medicine could be practiced individually and independently from others. The ultimate purpose of practice of medicine is towards service to mankind both alive and dead. A healthy relationship between Forensic Medicine and other clinical disciplines leads to a mutually rewarding situation. This paper outlines such clinic-pathologic relationships between forensic medicine and other clinical disciplines for better patient care in Sri Lanka.
\end{abstract}

\section{INTRODUCTION}

Sri Lanka is a country where there is a reasonably satisfactory coverage of medico-legal works with bare minimal resources and facilities. When compared with other disciplines of medicine whose primary aim is treating live patients and therapeutic diagnosis of illnesses, one can observe an immense difference in the context of resources and facilities available for these therapeutic disciplines of medicine and for forensic medicine. Still, the medico-legal services of the country are provided to a reasonable standard (as expressed by local specialists as well as foreign authorities who have been maintaining a close rapport with the medico-legal system of the country) using the knowledge, analytical capacity and anecdotal experiences of the forensic practitioners as well as the basic facilities such as H\&E staining of histopathological slides, limited facilities available at the Medical Research Institute, limited toxicology and odontology facilities available at the Institute of Legal Medicine and Toxicology-Colombo and the basic plain radiographic facilities reluctantly and remorsefully provided by the respective hospitals. This situation in forensic medicine in the second decade of the $21^{\text {st }}$ century could well be sighted similar to the situation of clinical medicine in the dawn of the $20^{\text {th }}$ century where physicians utilized the fundamentals of clinical examination such as inspection, palpation, percussion and auscultation with their treasured clinical experiences and basic and limited investigative facilities such as full blood count, urine full report and Rontgenographic studies so as to arrive at an accurate clinical diagnosis. In this context, the authors wish to explain how a bi-directional gain could be achieved ultimately aiming at better patient care, through developing a healthy relationship between Forensic Medicine and other clinical disciplines.

\section{DISCUSSION}

Clinical Forensic Medicine deals mainly with assaults, different types of accidents, sexual offenses, child abuse, domestic violence, consumption of alcohol and other drugs, torture and human rights violation, war-crimes, impaired motorists, deliberate self harm and so on whilst the diverse medico-legal issues revolving around a death is the arena of Forensic Pathology. Both in Clinical Forensic Medicine and in Forensic Pathology, the forensic practitioner may seek assistance and advice from the respective clinician to clarify certain issues and to maintain the accuracy and precision of his job. On the other hand, the forensic practitioner too can render a service to the clinician by enlightening him on certain twilight areas so as to rectify his clinical practice in future.

It is the duty of the first contact medical officer at the Out Patient Department or the Emergency Treatment Unit to objectively mention about the alcohol status of the patients admitted following assaults or traffic accidents. If he has failed to do so, then it becomes the duty of the houseman to assess and mention the same on the Bed Head Ticket (BHT). In practical context, this 
is the exception rather than the rule. Most of the times, nothing is mentioned about the alcohol status while in rare situations something like "alcohol++" is mentioned. It is only extremely rarely that one sees an objective assessment of alcohol status mentioned on the BHT by an admitting officer or a houseman. Not mentioning anything about alcohol status does not mean that the patient had been negative for alcohol but it only implies that the doctor has not taken any interest or attempt to inquire about the alcohol status of his patient. If this becomes a materially significant issue in the courts, then the doctor will have to answer for himself. The Judicial Medical Officer (JMO), to whom the patient is referred at a later date-possibly just prior to being discharged, will invariably be dependent on the BHT for filling the alcohol status on the Medico Legal Examination Form (MLEF).

In case of alleged sexual offenses and abortions, the clinician should not rush to interpret genital findings if he is not absolutely certain of the accuracy of his interpretations. There have been many instances where interpretation or the description given by the clinician on the BHT differs from that of the JMO invariably weakening the case before the courts. A common example would be misinterpretation of natural hymenal folds,notches or bumps as evidence of previous penetration. At the same time, everybody agrees that any forensic considerations are secondary to life saving measures. As such, if a clinician makes any therapeutic interventions on such a patient as surgical repairs of $3^{\text {rd }}$ degree perennial tears etc, it is prudent for him to attempt to take forensically important samples from the genital areas (such as vulvae, low-vaginal and high-vaginal swabs) and at least roughly describe the original injuries before such intervention is made. The same applies to preservation of clothing-specially the undergarments and the material recovered from the genital tract during the surgery. Informing the JMO as early as possible will lessen the medicolegal burden of the clinician as well as allow the JMO to carry out a more rewarding task.

In cases of suspected poisoning, it is prudent to inform the hospital police and then the JMO for further medico-legal management, even if the poisoning initially seems to be a selfingestion. The samples recovered from the patient such as vomitus, gastric-larvage, contaminated clothing, any suspected material handed over by the relatives and by-standers, initial blood and urine samples etc, should be properly stored and handed over to the JMO for further investigation, maintaining the chain of custody. It is also worth mentioning on the BHT about any peculiar clinical finding such as the smell of breath, colour, smell and the nature of gastric larvage, pupilary size, frothing, involuntary passage of stools and so on.

Burns are yet another type of injuries that lead to complicated medico-legal issues both in the survivors as well as in the dead. Delayed presentation to the JMO together with lack of interest by the clinicians to describe the forensically significant features such as the distribution, depth, surface area, drip marks, association with the clothing, any smell of accelerants such as kerosene, features peculiar to different types of acids and alkali, features suggesting the circumstances etc, make it a rather unrewarding exercise for the JMO to examine a burnt patient or to perform an autopsy on a burnt body. Further alteration of burns due to infection and surgical interventions such as escharectomy will further worsen the situation for the JMO.

We are living in an era of blood-borne infections such as HIV and Hepatitis B. It is the international practice that every autopsy be considered as high risk and thus to stick to universal precautions. Yet, it should be honestly accepted that we are performing in a sub-standard way in our busy mortuaries. It is the mortuary attendant/labourer who is at the highest risk. Therefore, it is vital that the clinicians convey the message of the infectious status of the deceased to the JMO, or at least the suspicion of such a situation prior to the autopsy so that additional precautions could be taken by the mortuary staff. In the same way, if the clinician suspects of such a condition, he should promptly investigate for the same after obtaining the written informed consent from the patient rather than expecting an answer from the forensic pathologist after the autopsy, who will have to depend on a less sensitive post mortem blood sample ${ }^{1}$.

In some occasions, patients tend to affirm that they do not wish to proceed for further legal actions and as such they are discharged from the wards without being seen by a JMO. There are 
hidden pitfalls in this practice. A person who has sustained injuries while committing a crime may "cook-up" a story of an innocent domestic accident and get away without being noticed by the law enforcement authorities. Clinicians, though they are quite capable of therapeutic management of the injuries, will be quite ignorant in recognizing the peculiar pattern of injuries standing itself as a proof of the fowl-play. On the other hand, a person who initially does not wish to go for legal actions, might later change his mind when he needs to obtain his personal insurance or when the other party who has initially promised him of a pecuniary reward following a traffic accident later declines to do so. Therefore it is prudent for the ward staff to hand over the responsibility to the JMO who has a better overall understanding about such circumstances.

Contribution of pre-existing natural illnesses to death in a person who has recently sustained trauma is always a dilemma for the forensic pathologist. Trauma or natural illness can separately and individually cause the death, while trauma can exacerbate the natural pathology and vice versa. A known patient with significant ischaemic heart disease, meeting with a traffic accident warranting a surgery and subsequently developing an acute myocardial infarction leading to his death is one such situation. Pulmonary thrombo-embolism, sub-dural haemorrhages etc are few other examples. In these conditions, a chronologically recorded, detailed and extensive history of the clinical presentations and the investigations done, would greatly help the pathologist to overcome the difficulties at least to a certain extent.

Injuries are surgically altered in numerous ways during clinical intervention. Trimming of the edges of an irregular laceration before suturing, painting with povidone iodine or Jansen violet, removal of foreign matter, shaving of head hair, application of bandages and plaster casts, insertion of intercostals tubes, drains and catheters, making surgical incisions, craniotomy and etc are just some examples. These are only to be expected as saving the life of the patient is supreme. Yet, sketching of the original wound, writing up of accurate surgical "OP" notes, taking foreign material recovered during the surgery in to safe custody etc can greatly help the JMO to "visualize" the original injury even though it might impose certain additional work load upon a busy houseman.

Due to invariable delay in the process of execution of justice, court cases will come up years after the initial management of the patient. Though rarely, sometimes it becomes pertinent to summon the clinician, the houseman or the staff nurse who was on duty in a particular ward on a particular day. Therefore, it is advisable to legibly mention the full name and the designation of the doctor or the subordinate ward staff member when making entries on the BHT. It is equally important to maintain a well-updated registry in each ward in every hospital over the years so that the ward staff who were on duty at a particular time on a particular day could be traced even years later.

An autopsy is the final systematic scientific investigation in to a death. The clinicians should understand the benefit of performing an autopsy, both for them as well as for the health care system in the country, in addition to the benefits to the deceased person and his immediate family members. A clinico-pathologic co-relation developed through a practice of performing high quality autopsies and comparing the findings with clinical data itself provides a clinical audit and an opportunity for self-rectification.

The autopsy rates are declining globally over the past few years, except in very few countires. ${ }^{2,3 .}$ It is a well known fact in the forensic literature that even the coroners and medical examiners tend to make mistakes in the cause and manner of death in the absence of an autopsy. A survey done in the Department of Forensic Medicine in Sydney, Australia had retrospectively reviewed investigative information of all cases in a six month period (429 cases) which were initially considered to be natural in circumstances. The authors have compared the presumptive causes of death with the actual post mortem findings. There were striking differences between the presumed cause of death and actual cause of death. The presumed cause of death was completely wrong in $28 \%$ of cases. There were several incidences where the manner of death which was originally thought to be natural had turned out to be non-natural at the autopsy. This highlights the importance of a good quality postmortem examination. ${ }^{3}$

Until around the sixth decade of the $20^{\text {th }}$ century, it was considered that performing an 
autopsy was crucial in cross-checking the clinical diagnosis as well as in understanding the diseases ${ }^{4}$. The advent of various imaging techniques and the "faith" the clinicians have vested upon these techniques is one reason for the falling autopsy rate. Several surveys in various countries have shown that the causes of death issued by physicians, even in hospital deaths bear an error rate of around $25-50 \%$. Correct cause of death is essential for policy planning at national level.

Results of a pilot study conducted in England and Wales aimed at improving the scrutiny of doctors revealed that the cause of death records are erroneous in one out of four patients ${ }^{5}$ and it highlights the importance of the autopsies in providing with a rather realistic picture of existing conditions.

Sensitivity and specificity of a test is a crucial matter, as certain lesions or conditions may not be accurately illustrated by investigative procedures such as post-mortem Computerized Tomographic (CT) scans and Ultra Sound scans (USS). Such findings could also be easily misinterpreted by a hospital/clinical radiologist who is not used to "reading" post-mortem conditions. Electrocardiography (ECG) does not always show certain disease conditions of heart. Authors have come across contradicting clinical findings with the post mortem findings of USS, $\mathrm{CT}$ scans and $\mathrm{ECG}^{6}$ etc which were mentioned on the BHT. Achieving of good quality reliable result depends on a good quality post mortem. For this purpose assistance of the clinicians, is really essential. It is a right of the next of kin to know the exact cause of death of their loved ones. It is not only a right, but also it helps to avoid wide array of misdiagnoses as well. When the policy makers do not get the accurate picture of why people have died, it invariably affects adversely on the future health policy planning of the country.

A seemingly natural death to a clinician could be a homicide or an occupationally related disease to the eye of the forensic pathologist as he always tries to consider the totality of circumstances ${ }^{7}$.

Finally, a healthy and mutually respectful relationship between the clinician and the forensic practitioner would invariably be helpful to both parties to improve the quality of their respective practices and above all will be a positive step towards achieving a best possible care for the patient and providing the justice for the deceased, while helping the accurate policy decisions of the country in the broadest context.

\section{References}

1. $\quad$ http://rethinkingaids.com/quotes/testpostmortem.html

2. The decline of the hospital autopsy: a safety and quality issue for healthcare in Australia. Med JAust. Mar 15 2004;180(6):281-5.

3. Nashelsky MB, Lawrence $\mathrm{CH}$. Accuracy of cause of death determination without forensic autopsy examination. Am J Forensic Med Pathol. 2003 Dec;24(4):313-9.

4. Roulson J, Benbow EW, Hasleton PS. Discrepancies between clinical and autopsy diagnosis and the value of post mortem histology; a meta-analysis and review. Histopathology. 2005 Dec;47(6):551-9.

5. Saukko P, Knight B. Knight's Forensic pathology. $3^{\text {rd }}$ edition. Edward Arnold:UK;2004.

6. Autopsy Rate and Physician Attitude Towards Autopsy: Elizabeth C Burton, Kim A Collins.

7. Jason Payne-James, Anthony Busuttil, William S. Smock. Forensic Medicine: Clinical and Pathological Aspects, Cambridge University Press, 2003. 